\title{
Estudio de capacidad de acogida del suelo en el Corredor Biológico Interurbano Río Torres, Costa Rica
}

\author{
Study of soil bearing capacity in the Río Torres Interurban \\ Biological Corridor, Costa Rica.
}

\author{
Osvaldo Navarro-Ceciliano ${ }^{1}$ \\ Instituto Tecnológico de Costa Rica; Cartago, Costa Rica
}

\begin{abstract}
Resumen
La capacidad de acogida del suelo en el Corredor Biológico Interurbano Río Torres se calculó por medio de tres variables superficiales (pedregosidad, profundidad efectiva y pendiente), el resultado mostró que las categorías de agricultura sin limitaciones y tierras forestales para protección son las más predominantes. Seguidamente, se definió el uso del suelo en el corredor a través de la clasificación supervisada de imágenes satelitales, donde 53,62\% pertenece al uso zonas urbanizadas, $25,7 \%$ a cobertura forestal, $19,91 \%$ a ganadería y pastos. Los resultados de capacidad de acogida y uso del suelo permitieron identificar las áreas con uso óptimo y sobreuso, estas últimas representan un $15,21 \%$ del corredor, para las cuales se le definieron buenas prácticas relacionadas al sector forestal, buscando disminuir la degradación del suelo. Por último, se evaluó la fragmentación de la cobertura forestal, demostrando que existe un alto nivel de segmentación, por lo que se recomendaron estrategias para restablecer la conectividad entre los fragmentos aislados.
\end{abstract}

Palabras clave: corredor biológico interurbano, capacidad de acogida del suelo, uso del suelo, fragmentación de la cobertura forestal.

\begin{abstract}
The soil bearing capacity in the Río Torres Interurban Biological Corridor was estimated employing three surface variables (stoniness, effective depth, and slope), the result indicated unrestricted agricultural and conservation forestland as the predominant categories. Next, land use types existing throughout the corridor were identified by means of the supervised classification of satellite images, which evidenced $53.62 \%$ of total area as occupied by urban activities, $25.7 \%$ forest coverage and
\end{abstract}

1 Ingeniero Forestal, Instituto Tecnológico de Costa Rica, Cartago, Costa Rica;

Correo electrónico: ognc110@gmail.com (DD https://orcid.org/0000-0002-7637-9577 
$19.91 \%$ destined to livestock grazing and pastures. Soil bearing capacity data and land use visualization allowed the identification of areas with optimal and overexploitation, the latter amounted to $15.21 \%$ of the corridor's total land area. For both types of areas, good forestry practices were defined in order to reduce soil degradation. Finally, forest coverage fragmentation was evaluated and found to exhibit a high degree of segmentation, thus strategies were recommended to reestablish connectivity between isolated fragments.

Keywords: Interurban biological corridor; Soil bearing capacity; Land use; Forest coverage fragmentation.

\section{Introducción}

La Compañía Nacional de Fuerza y Luz (CNFL) administra nueve plantas hidroeléctricas en el país, una de estas es la Planta Hidroeléctrica Belén (PHB), la cual fue instalada en 1914 sobre el río Virilla, con una potencia de $2500 \mathrm{~kW}$ (ICE, 2019); esta compañía se ha visto obligada a frenar la producción de esta y otras plantas en repetidas ocasiones, con el fin de extraer una gran cantidad de sedimentos que se acumulan en sus embalses, esto provoca grandes pérdidas económicas y además reduce la vida útil de los proyectos (Casamor y Calafat, 2018, párr. 6).

El manejo adecuado de las microcuencas es una alternativa que busca reducir el impacto causado por los residuos que llegan a los embalses, por este motivo la CNFL tomó la iniciativa de instaurar un Corredor Biológico Interurbano (CBI) sobre la microcuenca del río Torres que abastece al embalse de la PHB (Trujillo-Acosta, Peraza-Estrella, Marina-Hipólito y Feoli, 2016, p. 59).

El Reglamento a la Ley de Biodiversidad (2008) define el Corredor Biológico (CB) como un territorio delimitado geográficamente, con la finalidad de permitir conectividad entre distintos hábitats naturales o intervenidos, siendo su propósito principal el conservar y proteger la biodiversidad presente.

A partir de este concepto se crea la nueva modalidad de CBI, el cual hace referencia a un área urbana delimitada que permite la conectividad entre paisajes, ecosistemas y hábitats que han sido severamente modificados, con la finalidad de contribuir en la conservación de la biodiversidad (SINAC, 2018, p. 106).

La estrategia de conservación por corredores biológicos (CB) funciona como base para el manejo sostenible de los recursos naturales en 
cuencas hidrográficas, debido a que esta última no encierra la idea de homogeneidad como si lo hacen los CB (Feoli-Boraschi, 2012, p. 2).

Según lo mencionado por el comité local del Corredor Biológico Interurbano Río Torres (CBIRT), integrado por la CNFL, Municipalidad de San José, UCR, SINAC, entre otras organizaciones públicas, la última fase para instaurarlo requiere la elaboración de un estudio de la capacidad de acogida del suelo para determinar el estado de este recurso (Proyecto Biodiver_City San José, 2020).

En la teoría el concepto de capacidad de acogida hace referencia al mejor uso que se le pueda dar a un área basado en su sostenibilidad (Galacho y Arrebola, 2013, p. 70), por lo que un estudio de la capacidad de acogida del suelo analiza las propiedades de un territorio con el objetivo de definir si dicha área es óptima para el desarrollo de alguna actividad, además, Acosta, Suango, Proaño y Zambrado (2016, p. 34) mencionan que la capacidad de acogida se entiende como el grado de compatibilidad del territorio y sus recursos para tolerar diversos tipos de usos del suelo.

Actualmente, el uso del suelo abarca diferentes actividades, como asentamientos humanos, actividades agrícolas, extracción de minerales, hábitat biológico de diferentes ecosistemas naturales, entre otros, sin embargo durante los últimos años el crecimiento exponencial de ciertas actividades ha aumentado la presión sobre este, debido a la necesidad de satisfacer la demanda de alimento y el crecimiento económico mundial (Viscarra et al., 2016, p. 198), esto ha provocado impactos negativos, principalmente al medio ambiente, por lo que las partes involucradas en el manejo del suelo deben estar informadas y poseer conocimientos sobre el uso óptimo que se le debe dar a este recurso (McDowell et al., 2018, p. 212).

Los Sistemas de Información Geográfica son una herramienta de gran importancia para la zonificación y clasificación de uso del suelo, a través de un análisis espacial multivariable se pueden obtener resultados con una alta confiabilidad del área de estudio, lo que permite la toma de decisiones más acertadas con el objetivo de mejorar su sostenibilidad y evitar un sobreuso (Thakkara, Desai, Patel \& Potdar, 2017, p. 79).

El uso óptimo del suelo toma en cuenta aspectos económicos, sociales y de conservación, debido a que estos deben permanecer en equilibrio para permitir su protección y sostenibilidad, sin afectar el crecimiento económico de la sociedad; por lo que es de gran importancia definir el uso 
adecuado y correcto de este recurso (Adams, Pressey, \& Álvarez-Romero, 2016, p. 2) (Arjomandi, Mortazavi, Khalilian \& Garizi, 2021, p. 2).

Para los casos en los que existe un sobreuso del suelo debido al desequilibrio de alguno de los aspectos ya mencionados, por ejemplo, cuando el factor económico posea mayor influencia, se tenderá a realizar prácticas más intensivas de uso provocando degradación (Zweifel, Meusburger \& Alewell, 2019, p. 1), para esto es fundamental implementar buenas prácticas de uso del suelo en sitios con mayor afectación o sobreuso, buscando mejorar su conservación, además, generar beneficios en la protección de la biodiversidad existente y elevar la calidad de vida de los habitantes de la zona.

Existe una amplia gama de buenas prácticas para el manejo y uso sostenible del suelo, entre ellas destacan las relacionadas al sector forestal, tales como planes de restauración de la flora, manejo de áreas protegidas, reforestación, entre otras, estas prácticas son de gran importancia, debido a que no solo protegen el suelo, sino que también crean la posibilidad de restablecer la conectividad entre las áreas verdes que se encuentren fragmentadas o aisladas (FAO, 2018, p. 70).

La fragmentación se define como la transformación de un hábitat continuo a uno segmentado, causado generalmente por la necesidad de establecer nuevos usos del suelo, esto genera fragmentos aislados que presentan condiciones diferentes con respecto al hábitat original, afectando la abundancia y diversidad de especies, así como las relaciones ecológicas existentes, por lo que recuperar la conectividad de estas áreas aisladas es fundamental para la permanencia y protección de la biodiversidad (Luther et al., 2020, p. 2).

Por ende, la finalidad del proyecto es realizar el estudio de capacidad de acogida del suelo en el CBIRT para identificar las zonas que presenten condiciones de sobreuso, y a partir de esto, recomendar la implementación de diferentes actividades relacionadas al sector forestal con el objetivo de mejorar su protección y conservación, además de establecer estrategias de conectividad entre las áreas verdes aisladas, buscando aumentar la biodiversidad del área. 
Osvaldo Navarro-Ceciliano

Estudio de capacidad de acogida del suelo en el Corredor Biológico Interurbano Río Torres, Costa Rica

\section{Materiales y métodos}

\section{Descripción del área de estudio}

El CBIRT se encuentra dentro de la subcuenca del Río Virilla, posee una longitud de $26 \mathrm{~km}$ y un ancho de 300 metros a ambos lados del Río Torres, el área total del corredor es de 4309 hectáreas. Este estudio abarcó parte de los cantones de Goicoechea, Montes de Oca, San José y Tibás (Trujillo-Acosta et al., 2016, p. 55).

La cobertura predominante en el corredor es la urbana, la misma presenta un crecimiento poco planificado, otras coberturas presentes son: bosque, agricultura y pastos. La elevación promedio es de 1100 metros sobre el nivel del mar y la densidad promedio poblacional es de 5112 habitantes por km² (Pérez-Gómez, Gastezzi-Arias y Vega-Quesada, 2016, p. 21). En la figura 1 se muestra la extensión y ubicación del CBIRT.

Figura 1. Mapa de ubicación el área de estudio, CBIRT.

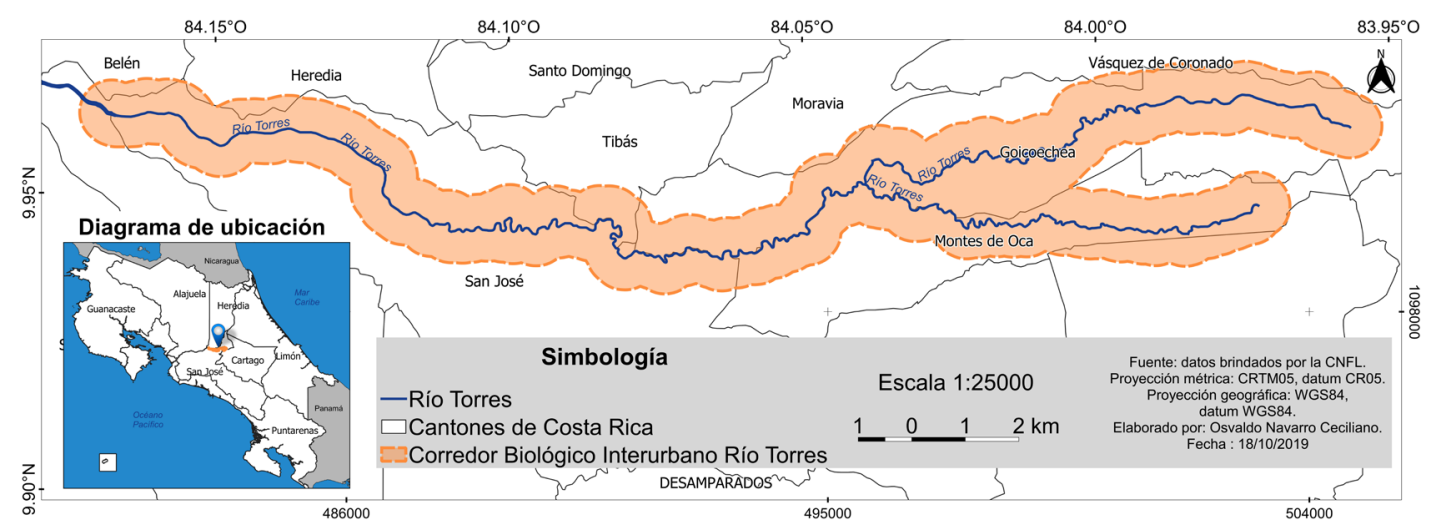

\section{Capacidad de acogida del suelo}

Por solicitud expresa de la CNFL se utilizó la metodología de Gómez, elaborada en Ecuador, para determinar la capacidad de acogida del suelo en el CBIRT, debido a que por hábito y otros motivos la compañía ha trabajado con esta durante los últimos años, causa que justifica el no haber empleado la metodología establecida como obligatoria para Costa Rica mediante el decreto $\mathrm{N}^{\circ}$ 41960-MAG-MIRENEM (Metodología Determinación Capacidad Uso Tierras Costa Rica, 2019). 
La metodología establece varias categorías de capacidad de acogida del suelo como se muestra en el cuadro 1, las cuales van desde las más permisivas hasta las más restrictivas de uso.

Cuadro 1. Categorías de capacidad de acogida del suelo según Gómez (2014, p. 79-80).

\begin{tabular}{|c|c|c|}
\hline \multirow{8}{*}{ 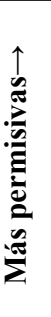 } & Categorías & \multirow{8}{*}{$\begin{array}{l}3 \\
0 \\
0 \\
0 \\
0 \\
0 \\
0 \\
0 \\
0 \\
0 \\
\vdots \\
\downarrow \\
1\end{array}$} \\
\hline & Agricultura sin limitaciones (A) & \\
\hline & Agricultura con mejoras (Am) & \\
\hline & Agroforestería con cultivos anuales (Aa) & \\
\hline & Sistemas silvopastoriles $(\mathrm{Ss})$ & \\
\hline & Agroforestería con cultivos permanentes (Ap) & \\
\hline & Tierras forestales para producción $(\mathrm{F})$ & \\
\hline & Tierras forestales de protección $(\mathrm{Fp})$ & \\
\hline
\end{tabular}

Gómez (2014, p. 77) define como factores determinantes la profundidad efectiva del suelo y la pendiente del terreno, adicionalmente, se considera la pedregosidad (superficial e interna) y el drenaje superficial como factores que en forma temporal o permanente pueden modificar la capacidad de acogida del suelo.

La CNFL brindó la información de las variables profundidad efectiva del suelo y pedregosidad, adicionalmente, la variable pendiente se estimó mediante un modelo de elevación digital. Sin embargo, la variable drenaje superficial no se consideró en este estudio, ya que no se contaba con los datos correspondientes y según lo mencionado por Sánchez, Valle, Scorza, Feoli y Artavia (2015, p. 24), la misma no influye en la limitación de la capacidad de acogida por las características de relieve que posee el área. Al obtener las variables se procedió a trabajar con las matrices de decisión y asignación de categorías.

En el cuadro 2 se muestra la matriz que combina los factores profundidad del suelo y pendiente, lo que permitió la asignación del área de estudio entre diferentes categorías, cuando se consideró más de una categoría posible, se dio prioridad a la de menor intensidad de uso.

En el cuadro 3 se muestra la modificación de la capacidad de acogida según el nivel en que se presenta el factor modificador de pedregosidad, en este se excluyó el factor limitante de drenaje por las razones explicadas anteriormente. 
Cuadro 2. Matriz de capacidad de acogida del suelo según la metodología de Gómez (2014, p. 81).

\begin{tabular}{|c|c|c|c|c|c|}
\hline \multirow{2}{*}{$\begin{array}{c}\text { Profundidad del } \\
\text { suelo (cm) }\end{array}$} & \multicolumn{5}{|c|}{ Pendientes (\%) } \\
\cline { 2 - 6 } & $0-12$ & dic- 25 & $25-50$ & $50-70$ & $>70$ \\
\hline$>90$ & $\mathrm{~A}$ & $\mathrm{Am} / \mathrm{Aa}$ & $\mathrm{Ap} / \mathrm{F}$ & $\mathrm{F} / \mathrm{Fp}$ & $\mathrm{Fp}$ \\
\hline $50-90$ & $\mathrm{~A}$ & $\mathrm{Am} / \mathrm{Aa}$ & $\mathrm{Ap} / \mathrm{F}$ & $\mathrm{F} / \mathrm{Fp}$ & $\mathrm{Fp}$ \\
\hline $20-50$ & $\mathrm{Am} / \mathrm{Aa}$ & $\mathrm{Aa} / \mathrm{Ss}$ & $\mathrm{Ap} / \mathrm{F}$ & $\mathrm{F} / \mathrm{Fp}$ & $\mathrm{Fp}$ \\
\hline$<20$ & $\mathrm{Am} / \mathrm{Aa}$ & $\mathrm{Ss}$ & $\mathrm{Fp}$ & $\mathrm{Fp}$ & $\mathrm{Fp}$ \\
\hline
\end{tabular}

Cuadro 3. Modificaciones a las categorías de capacidad de acogida en función de la pedregosidad según la metodología de Gómez (2014, p. 81).

\begin{tabular}{|c|c|c|}
\hline Categoría sin modificar & Pedregosidad & Categoría modificada \\
\hline \multirow[t]{2}{*}{$\bar{A}$} & No limitante & $\mathrm{A}$ \\
\hline & Limitante & Ss \\
\hline \multirow[t]{2}{*}{$\mathrm{Am}$} & No limitante & $\mathrm{Am}$ \\
\hline & Limitante & Ss \\
\hline \multirow[t]{2}{*}{$\mathrm{Aa}$} & No limitante & $\mathrm{Aa}$ \\
\hline & Limitante & Ss \\
\hline Ss & Limitante & $\mathrm{F} / \mathrm{Fp}$ \\
\hline \multirow[t]{2}{*}{ Ap } & No limitante & Ap \\
\hline & Limitante & $\mathrm{F} / \mathrm{Fp}$ \\
\hline \multirow[t]{2}{*}{$\mathrm{F}$} & No limitante & $\mathrm{F}$ \\
\hline & Limitante & $\mathrm{Fp}$ \\
\hline \multicolumn{3}{|c|}{$\begin{array}{l}\text { CASOS ESPECIALES: La categoría Ss por definición ya presenta limitaciones de pedregosida } \\
\text { y/o de drenaje, por lo que su grado de manifestación determina que permanezca como Ss o bien } \\
\text { se modifique hacia F o Fp. }\end{array}$} \\
\hline
\end{tabular}

Para obtener la capacidad de acogida del suelo, Gómez (2014, p. 80) define que es necesario contemplar las zonas de protección dentro del área de estudio, las cuales se incluyeron dentro de la categoría de tierras forestales de protección (FP), estas zonas corresponden a áreas de recarga facilitadas por el SENARA y áreas de protección aledañas a los cuerpos de agua (ríos, embalses y nacientes) establecidas según lo estipulado por la Ley Forestal 7575 (1996).

\section{Uso actual del suelo}

Se seleccionó una imagen satelital Landsat 8 de la página web del Servicio Geológico de los Estados Unidos (USGS) captada en febrero del año 2019, la cual posee una resolución espacial de 30 metros, además se 
encontraba georreferenciada y ortorectificada (Astola, Häme, Sirro, Molinier \& Kilpi, 2019, p. 258).

Finalmente, se le aplicó corrección atmosférica y el preprocesamiento por medio de programas de sistemas de información geográfica (SIG). Durante los meses de agosto y septiembre del mismo año se procedió a visitar los sitios considerados por expertos del comité local del CBIRT como claves para evaluar el uso del suelo, se tomaron puntos GPS de referencia en cada uno y se definió su uso según el Sistema de clasificación del uso y la cobertura de la tierra para Costa Rica (2018), ya que la metodología de Gómez no define un sistema para clasificación de uso del suelo, solo para la capacidad de acogida. Cada punto se utilizó como base para realizar la clasificación supervisada de la imagen satelital a través de programas de SIG.

La exactitud del resultado de la clasificación se validó mediante datos numéricos y estadísticos, por lo que se elaboró una matriz de confusión para comparar lo observado en imágenes satelitales de alta resolución con respecto al uso generado por medio de la clasificación supervisada, lo que permitió calcular el porcentaje de exactitud global del mapa de uso generado, además de la exactitud del clasificador y la exactitud del usuario, según como lo proponen Boca y Rodríguez (2012, p. 13-15).

Asimismo, se realizó un análisis de probabilidad mediante el estadístico Kappa a través de la fórmula 3.

$$
\mathrm{K}_{\mathrm{hat}}=\frac{\mathrm{N} * \sum_{\mathrm{i}=\mathrm{r}}^{\mathrm{r}} \mathrm{x}_{\mathrm{ii}}-\sum_{\mathrm{i}=\mathrm{r}}^{\mathrm{r}}\left(\mathrm{x}_{\mathrm{i}+} * \mathrm{x}_{+\mathrm{i}}\right)}{\mathrm{N}^{2}-\sum\left(\mathrm{x}_{\mathrm{i}+} * \mathrm{x}_{+\mathrm{i}}\right)}
$$

Donde:

r: número de filas en la matriz.

$\mathrm{X}_{\mathrm{ii}}$ : suma de observaciones en la fila i, y columna i (los valores en la diagonal).

$\mathrm{X}_{\mathrm{i}+}$ : total de observaciones en la fila $\mathrm{i}$ (el total en la fila i a la derecha de la matriz).

$\mathrm{X}_{+\mathrm{i}}$ : total de observaciones en la columna i (total en la columna $\mathrm{i}$ debajo de la matriz).

$\mathrm{N}$ : número total de observaciones o puntos de control usados en la validación. 
Para determinar si el uso del suelo en el CBIRT ha sufrido cambios durante los últimos años, se comparó el resultado generado con respecto a lo establecido por Sánchez, Valle-Hidalgo, Feoli y Murillo (2016, p. 39) para el año 2016.

\section{Sobreuso y uso óptimo del suelo}

A través de los resultados obtenidos en los procesos anteriores (capacidad de acogida y uso del suelo) se identificaron las áreas que se encuentran actualmente en sobreuso y las que poseen uso óptimo, por medio de la matriz elaborada para la identificación de estas áreas, como se muestra en el cuadro 4, la cual toma como referencia la capacidad de acogida del suelo con respecto al uso que posee actualmente el corredor, esto quiere decir que si un sitio es apto para establecer agricultura sin limitaciones (A), pero posee uso del suelo de cobertura forestal, se le clasificó como uso óptimo debido a que no existe riesgo de degradación; en caso contrario cuando un área es apta solo para tierras forestales de protección y posee uso de agricultura, se le definió como sobreuso del suelo, así se realizó por cada categoría y uso del suelo identificado.

Cuadro 4. Matriz para la identificación de las áreas con sobreuso (SU) y uso óptimo (UO), con respecto a las categorías capacidad de acogida determinada en el CBIRT.

\begin{tabular}{|c|c|c|c|}
\hline \multirow[b]{2}{*}{$\begin{array}{l}\text { Categorías de capacidad de acogida del suelo en } \\
\text { el CBIRT }\end{array}$} & \multicolumn{3}{|c|}{ Usos del suelo } \\
\hline & $\begin{array}{c}\text { Cobertura } \\
\text { forestal }\end{array}$ & \begin{tabular}{|c|} 
Ganadería y \\
pastos
\end{tabular} & $\begin{array}{c}\text { Agricultura } \\
\text { (café) }\end{array}$ \\
\hline Agricultura sin limitaciones (A) & $\mathrm{UO}$ & $\mathrm{UO}$ & $\mathrm{UO}$ \\
\hline Agricultura con mejoras (Am) & $\mathrm{UO}$ & $\mathrm{UO}$ & $\mathrm{UO}$ \\
\hline Agroforestería con cultivos anuales $(\mathrm{Aa})$ & UO & UO & UO \\
\hline Sistemas silvopastoriles (Ss) & UO & UO & UO \\
\hline Agroforestería con cultivos permanentes (Ap) & UO & SU & UO \\
\hline Tierras forestales para producción $(\mathrm{F})$ & UO & SU & SU \\
\hline Tierras forestales de protección $(\mathrm{Fp})$ & UO & SU & SU \\
\hline
\end{tabular}

Posteriormente, se establecieron recomendaciones de buenas prácticas de uso relacionadas al sector forestal para las áreas que presentaron problemas de sobreuso, además, es importante aclarar que la metodología 
de Gómez (2014, p. 77) establece que el uso del suelo identificado como "Zonas Urbanizadas" debe ser excluido del estudio, debido a que no se puede modificar o sustituir.

Sin embargo, como este representa un gran porcentaje del corredor, se decidió recomendar de igual forma buenas prácticas con el objetivo de aumentar la cobertura forestal y así lograr la conectividad a lo largo del mismo.

\section{Fragmentación de la cobertura forestal}

El grado de fragmentación de la cobertura forestal fue obtenido mediante la herramienta de SIG Guidos Toolbox, los criterios utilizados para definirlo toman en cuenta el tamaño del fragmento o parche y el nivel de conectividad con respecto a sus vecinos dentro del área de estudio, a partir de esto se definió a través de la herramienta cuatro grados de fragmentación, los cuales son insularizado, altamente fragmentado, fragmentación moderada y sin fragmentación (mencionados desde el más a menos afectado), para evaluar el estado en que se encuentra cada área verde y considerar el restablecimiento o no de la conectividad, tomando en cuenta el nivel de dificulta que esto conllevaría.

\section{Resultados y discusión \\ Capacidad de acogida del suelo}

En la figura 2 se presenta la distribución de las categorías de capacidad de acogida en el CBIRT, el área y el porcentaje que corresponde a cada categoría se muestra en cuadro 5.

Figura 2. Capacidad de acogida del suelo en el Corredor Biológico Interurbano Río Torres, San José, Costa Rica.

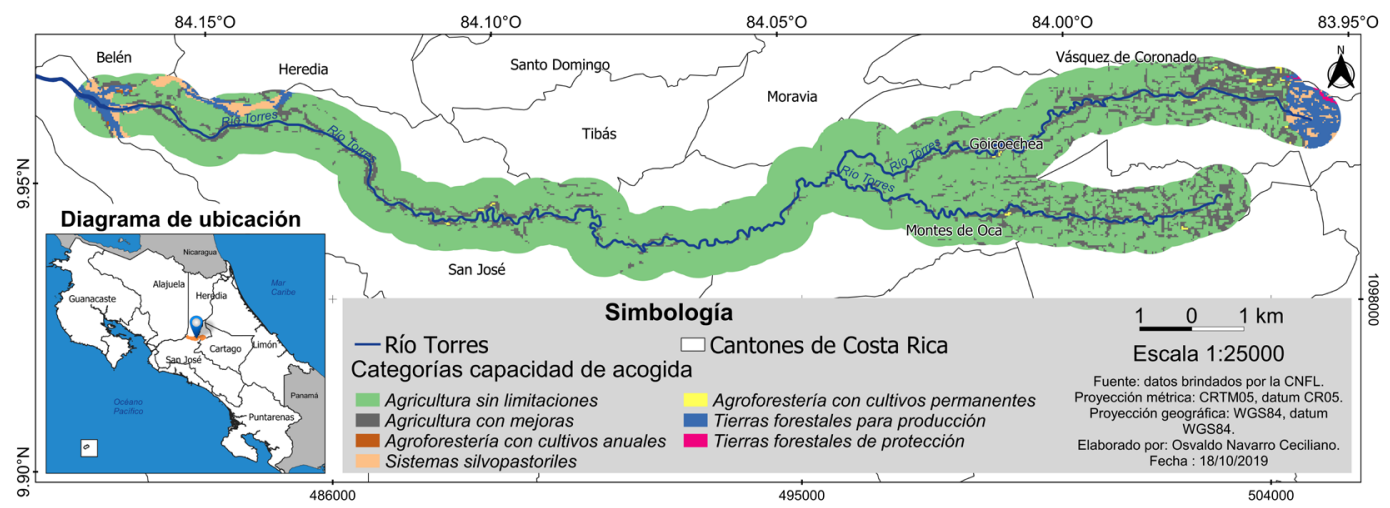


Osvaldo Navarro-Ceciliano

Estudio de capacidad de acogida del suelo en el Corredor Biológico Interurbano Río Torres, Costa Rica

Cuadro 5. Área en hectáreas y porcentaje de las categorías de capacidad de acogida del suelo.

\begin{tabular}{|c|c|c|}
\hline Categorías de capacidad de acogida & Área (ha) & Porcentaje (\%) \\
\hline Agricultura sin limitaciones & 3437,51 & 79,75 \\
\hline Agricultura con mejoras & 648,49 & 15,04 \\
\hline Agroforestería con cultivos anuales & 0,81 & 0,02 \\
\hline Sistemas silvopastoriles & 80,75 & 1,87 \\
\hline Agroforestería con cultivos permanentes & 6,84 & 0,16 \\
\hline Tierras forestales para producción & 131,97 & 3,06 \\
\hline Tierras forestales de protección & 4,23 & 0,1 \\
\hline
\end{tabular}

Según los resultados obtenidos de la capacidad de acogida del CBIRT, gran parte de este pertenece a la categoría agricultura sin limitaciones, lo cual indica que son sitios con aptitud para cultivos agrícolas (monocultivo o asociados), sin limitaciones por variables físicas y que demandan nulas o pocas prácticas de conservación de suelos (Gómez, 2014, p. 79).

Otra categoría con un grado significativo de representación es agricultura con mejoras, los sitios que pertenecen a esta presentan limitaciones de uso moderadas respecto a las variables físicas, por lo que para poder implementar actividades relacionadas a la agricultura es necesario establecer prácticas de manejo y conservación de suelos (Gómez, 2014, p. 79). Así mismo, solo un bajo porcentaje del corredor está representado por las tierras forestales de protección, lo cual indica que el área con limitaciones severas de uso del suelo es muy reducida.

Sin embargo, el resultado anterior no consideró las zonas de protección definidas por la legislación del país, al incluirlas se obtuvieron cambios en los porcentajes de las categorías de capacidad de acogida del suelo como se muestran en la figura 3 y el cuadro 6 , en estos se puede observar que el cambio más representativo fue para las tierras forestales de protección pasando de $0,1 \%$ a $54,2 \%$, seguido por agricultura sin limitaciones que se redujo a $41,44 \%$; las demás categorías presentan valores que oscilan entre 0,02 y $3,27 \%$. 
Figura 3. Capacidad de acogida del suelo considerando zonas de protección en el Corredor Biológico Interurbano Río Torres, San José, Costa Rica.

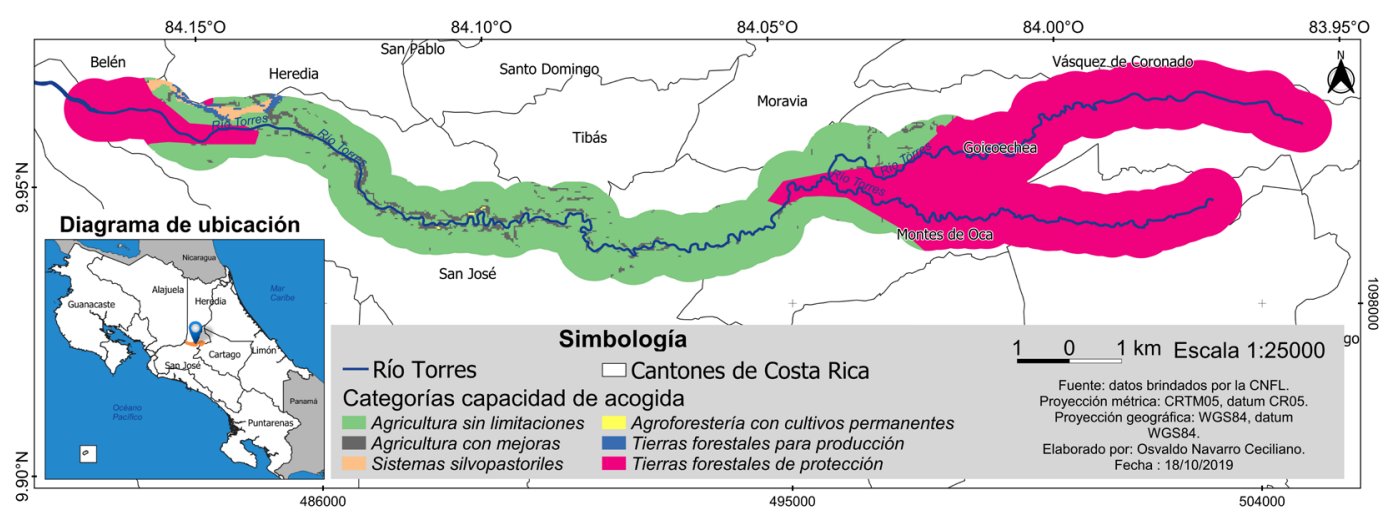

Cuadro 6. Área en hectáreas y porcentaje de las categorías de capacidad de acogida del suelo considerando zonas de protección.

\begin{tabular}{|c|c|c|}
\hline Categorías de capacidad de acogida & Área (ha) & Porcentaje (\%) \\
\hline Agricultura sin limitaciones & 1787.77 & 41.44 \\
\hline Agricultura con mejoras & 140.92 & 3.27 \\
\hline Sistemas silvopastoriles & 29.46 & 0.68 \\
\hline Agroforestería con cultivos permanentes & 0.98 & 0.02 \\
\hline Tierras forestales para producción & 16.77 & 0.39 \\
\hline Tierras forestales de protección & 2338.13 & 54.2 \\
\hline
\end{tabular}

El aumento en el porcentaje correspondiente a la categoría tierras forestales de protección refleja la importancia de tomar en cuenta las zonas de protección definidas por ley, debido a que estas son apropiadas exclusivamente para actividades forestales de conservación y limita cualquier cambio de uso; por otra parte, la categoría agricultura sin limitaciones sigue abarcando un gran porcentaje, estos valores indican que el corredor se encuentra principalmente categorizado en zonas con severas y reducidas limitaciones de uso 
Osvaldo Navarro-Ceciliano

Estudio de capacidad de acogida del suelo en el Corredor Biológico Interurbano Río Torres, Costa Rica

\section{Uso del suelo}

A partir de la clasificación supervisada se identificaron 5 clases de uso del suelo, como se muestran en la figura 4. Las zonas urbanizadas ocuparon el mayor porcentaje de uso con un 53,62\% (2310,85 ha), seguidas por la cobertura arbórea con un $25,7 \%$ (1107,6 ha), donde se incluyen bosques secundarios, áreas verdes en zonas urbanas y plantaciones forestales; la clase de ganadería y pastos ocupa el 19,91\% (857,91 ha) del corredor, en esta última incluye pastos arbolados, pastos limpios y charrales; por otra parte, en menor proporción se encuentran las clases agricultura y zonas húmedas con $0,75 \%(32,52$ ha) y $0,02 \%(0,99 \mathrm{ha})$ respectivamente.

Figura 4. Uso actual del suelo en el Corredor Biológico Interurbano Río Torres, San José, Costa Rica.

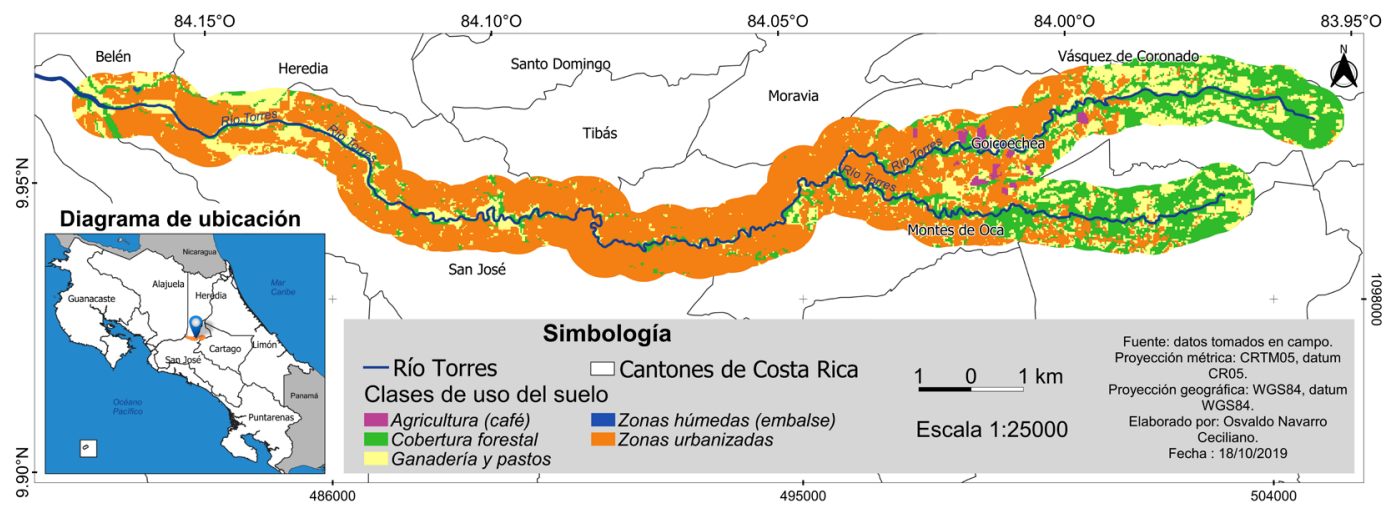

La clase Zonas Urbanizadas se concentra principalmente en la parte media y baja del área de estudio; en lo que respecta al bosque, la mayor presencia de este se encuentra en la parte alta del corredor e igual sucede con los pastos dedicados a la ganadería; por su parte, la actividad agrícola, representada por cultivos de café, solo se concentra en un área, esto coincide con lo mencionado por Trujillo-Acosta et al. (2016, p. 60).

Al comparar los resultados obtenidos con respecto a lo definido por Sánchez et al. (2016, p. 39) para el año 2016, se observa que la cobertura forestal ha disminuido al menos un $3 \%$, esto se debe principalmente a que existe presión sobre los bosques en las zonas altas del corredor, ya que se 
busca expandir los territorios dedicados a la ganadería y pastos, evidenciado por un aumento de $4 \%$ para esta clase.

Es importante destacar, que la clase agricultura ha presentado una reducción bastante considerable, pasando de $4,28 \%$ a $0,75 \%$, el principal motivo de esta situación es la caída del precio del café en el mercado internacional, provocando que la actividad sea poco rentable (Organización Internacional del café, 2019, p. 1) y, por lo tanto, en la actualidad estas zonas son sustituidas por uso urbano.

A través de una matriz de confusión se validó la clasificación (cuadro 7), en las filas se colocaron los datos clasificados y en las columnas los datos tomados de la imagen satelital, la diagonal gris muestra la cantidad de puntos que fueron correctamente clasificados por clase, a partir de esta se permitió demostrar el grado de exactitud obtenido de la clasificación, (cuadro 8), donde la exactitud global obtenida fue de 93,75\%, además, el valor del estadístico Kappa $\left(\mathrm{K}_{\text {hat }}\right)$ obtenido es de 0,9125.

Cuadro 7. Matriz de confusión entre la información de la imagen satelital Lansat 8 y los resultados de la clasificación de uso del suelo del CBIRT, San José, Costa Rica.

\begin{tabular}{|c|c|c|c|c|c|c|c|}
\hline & & \multicolumn{5}{|c|}{ Información de imagen satelital } & \multirow[b]{2}{*}{ Tota } \\
\hline & Clase & 1000 & 2000 & 3000 & 4000 & 5000 & \\
\hline \multirow{6}{*}{ 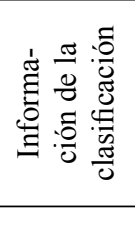 } & 1000 & 11 & & & & & 11 \\
\hline & 2000 & & 2 & & & & 2 \\
\hline & 3000 & & & 7 & & & 7 \\
\hline & 4000 & & & & 1 & & 1 \\
\hline & 5000 & 1 & & 1 & & 9 & 11 \\
\hline & Total & 12 & 2 & 8 & 1 & 9 & 32 \\
\hline
\end{tabular}

Cuadro 8. Exactitud de los resultados de la clasificación de uso del suelo del CBIRT, San José, Costa Rica.

\begin{tabular}{|c|c|c|c|c|c|}
\hline \multirow{2}{*}{ Exactitud } & \multicolumn{5}{|c|}{ Clase } \\
\cline { 2 - 6 } & $\mathbf{1 0 0 0}$ & $\mathbf{2 0 0 0}$ & $\mathbf{3 0 0 0}$ & $\mathbf{4 0 0 0}$ & $\mathbf{5 0 0 0}$ \\
\hline Del productor & 91,7 & 100 & 87,5 & 100 & 100 \\
\hline Del usuario & 100 & 100 & 100 & 100 & 81,81 \\
\hline General & \multicolumn{5}{|c}{} \\
\hline
\end{tabular}


La exactitud del productor o clasificador para las clases de agricultura (2000), zonas húmedas (4000) y zonas urbanizadas (5000) presentó una precisión de $100 \%$, ya que todas las unidades muestreadas fueron correctamente clasificadas con respecto a los datos de referencia.

Por otra parte, para la clase de cobertura forestal (1000) se obtuvo una exactitud de $91,7 \%$ y con una menor precisión la clase de ganadería y pastos $(87,5 \%)$, lo cual indica que solo un bajo porcentaje de las unidades muestreadas no concuerdan con los datos de referencia, respecto a la exactitud del usuario la clase de zonas urbanizadas (5000) presentó una precisión con un valor de $81,8 \%$, lo que significa que esta fue asignada de manera incorrecta en otros usos, además es importante destacar que las restantes clases obtuvieron un $100 \%$ de precisión.

La exactitud general o global es de $93,75 \%$, esto quiere decir, que la clasificación coincide en ese porcentaje con respecto a la realidad del uso actual del suelo en el corredor. Por otra parte, según el estadístico Kappa, la clasificación fue mejor en $91,25 \%$ que la realizada por el simple azar, siendo esta última similar a una clasificación visual en campo (Casco, Arias, Mareco y Kindgard, 2016, apartado 4.6 Medición de la confiabilidad de un mapa).

\section{Zonas de uso óptimo y sobreuso del suelo}

Al determinar las zonas de uso óptimo y sobreuso del suelo (figura 5), se obtuvo que 1352,31 ha $(31,59 \%)$ del corredor se encuentran en uso óptimo, mientras que 640,27 ha $(15,21 \%)$ se encuentran en sobreuso.

Además, en la figura 6 se identifican por categoría de uso del suelo los sitios que poseen sobreuso, las cuales fueron agricultura $(30,88 \mathrm{ha}) \mathrm{y}$ ganadería y pastos $(609,39$ ha). 
Figura 5. Determinación de las zonas que presentan sobreuso y uso óptimo del suelo en el Corredor Biológico Interurbano Río Torres, San José, Costa Rica.

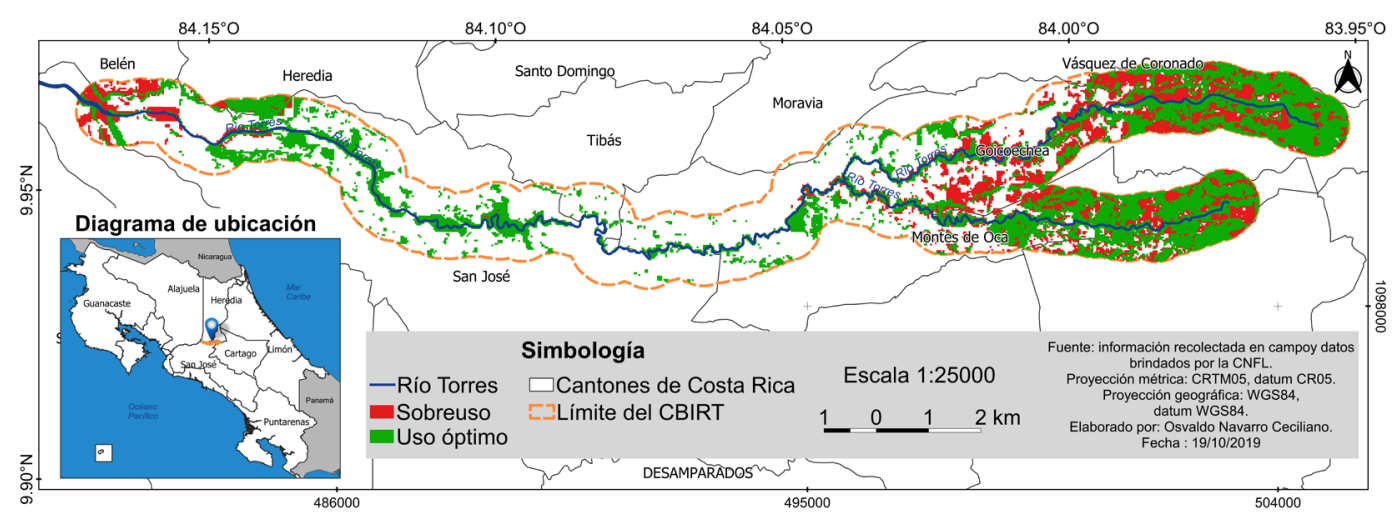

Figura 6. Uso actual incorrecto del suelo por categoría de uso en el Corredor Biológico Interurbano Río Torres, San José, Costa Rica.

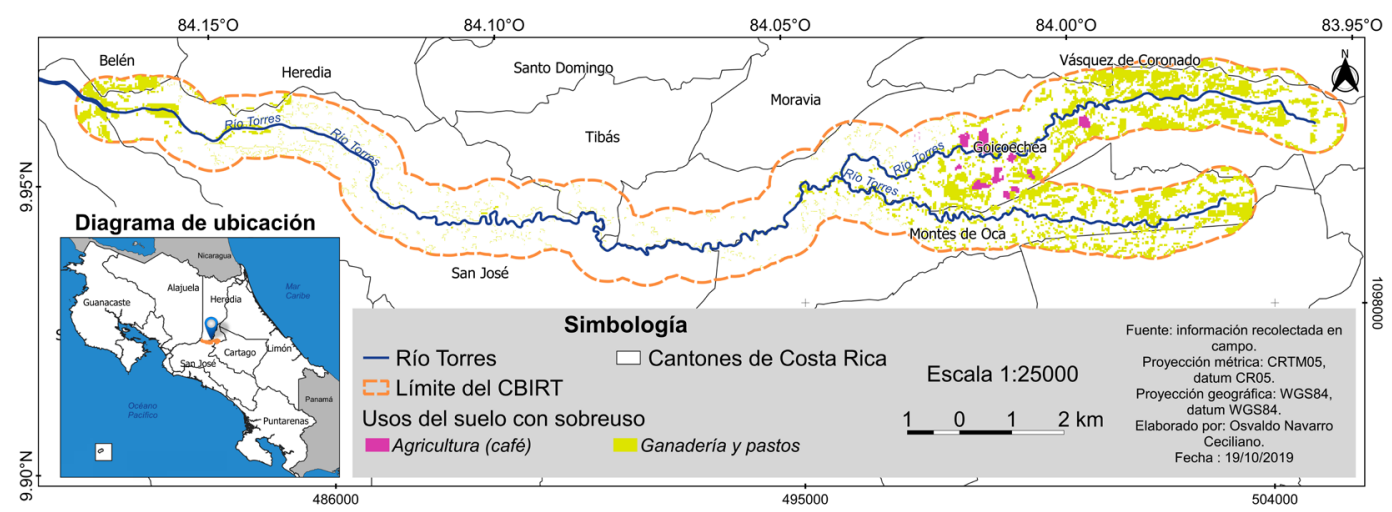

Un 15,21\% (640,27 ha) del área de estudio posee sobreuso por lo que es importante realizar actividades que mejoren su condición, tal como lo menciona Suryatmojo (2014, p. 833), con el fin de evitar daños que disminuyan las funciones y calidad del suelo.

Por lo que se recomiendan establecer actividades de restauración y reforestación, esto debido a que las raíces de los árboles ofrecen estabilidad y apoyo mecánico para impedir desprendimientos de tierra en sitios 
con altas pendientes o disminuir la pérdida de suelo por la escorrentía del agua (FAO, 2018, p. 86).

En el cuadro 9 se establecen las prácticas forestales más adecuadas por clase de uso para mejorar la protección del suelo en las zonas con sobreuso ya mencionadas.

\section{Cuadro 9. Definición de prácticas forestales para mejorar el uso del suelo en el Corredor Biológico Interurbano Río Torres.}

\begin{tabular}{|c|c|c|c|}
\hline $\begin{array}{c}\text { Tipo de } \\
\text { uso }\end{array}$ & $\begin{array}{l}\text { Actividad } \\
\text { propuesta }\end{array}$ & Procedimiento y recomendaciones & Especies recomendadas \\
\hline 莺 & $\begin{array}{l}\text { Implementar sis- } \\
\text { tema agro-forestal } \\
\text { (SAF). }\end{array}$ & $\begin{array}{c}\text { Se debe definir los objetivos del SAF, } \\
\text { identificar las características del sitio y } \\
\text { seleccionar especies con valor comercial, } \\
\text { que se desarrollen adecuadamente en las } \\
\text { condiciones de cada lugar, así mismo se } \\
\text { deben planificar prácticas de manejo de } \\
\text { los árboles seleccionados. En combinación } \\
\text { con agricultura se recomienda establecer } \\
\text { cortinas rompevientos, plantar árboles en } \\
\text { asociación a los cultivos o establecer cercas } \\
\left.\text { vivas (Decreto } \mathrm{N}^{\circ} 23214,1994\right) \text {. } \\
\end{array}$ & $\begin{array}{l}\text { Cordia alliodora, Gliricidia } \\
\text { sepium, Inga sp, Bursera sima- } \\
\text { ruba, Tabebuia impetiginosa, } \\
\text { Tabebuia ochracea. }\end{array}$ \\
\hline \multirow{2}{*}{ 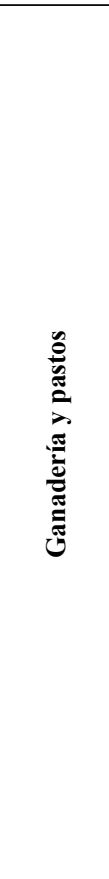 } & $\begin{array}{c}\text { Restaurar las áreas } \\
\text { degradadas de las } \\
\text { riberas de ríos. }\end{array}$ & \begin{tabular}{|c|} 
El porte máximo sugerido para los indi- \\
viduos debe ser menor a $15 \mathrm{~m}$. Lo ideal \\
es que sean árboles con buen atractivo, \\
tanto en flores o frutos (Subcomisión \\
Heredia - CGICRG Tárcoles, sf, p. 33). \\
Es importante considerar que los árboles \\
se pueden encontrar expuestos a daños \\
por el pastoreo del ganado, para estos \\
casos es recomendable limitar el acceso \\
a los animales en estas áreas (Decreto \\
$\left.\mathrm{N}^{\circ} 23214,1994\right)$. \\
\end{tabular} & $\begin{array}{c}\text { Luehea speciosa, Ochroma } \\
\text { pyramidale, Stemmadenia } \\
\text { donnell-smithii, Urera sp, } \\
\text { Bursera simaruba, Gliricidia } \\
\text { sepium, Cecropia } \mathrm{sp} \text {, Inga } \mathrm{sp}, \\
\text { Zygia longifolia, Clusia rosea, } \\
\text { Ficus sp, Guazuma ulmifoli, } \\
\text { Cassia grandis, Acnistus } \\
\text { arborescens. }\end{array}$ \\
\hline & $\begin{array}{c}\text { Implemen-tar siste- } \\
\text { ma agro-forestal } \\
\text { (SAF) }\end{array}$ & $\begin{array}{c}\text { Se debe definir los objetivos del SAF, } \\
\text { identificar las características del sitio y } \\
\text { seleccionar especies con valor comercial, } \\
\text { que se desarrollen adecuadamente en las } \\
\text { condiciones de cada lugar, así mismo se } \\
\text { deben planificar prácticas de manejo de } \\
\text { los árboles seleccionados. En combina- } \\
\text { ción con ganadería y pastos se recomien- } \\
\text { da establecer sistemas silvopastoriles, } \\
\text { cortinas rompevientos. Es importante } \\
\text { considerar que los árboles se pueden en- } \\
\text { contrar expuestos a daños por el pastoreo } \\
\text { del ganado, para estos casos es recomen- } \\
\text { dable limitar el acceso a los animales en } \\
\left.\text { estas áreas (Decreto } \mathrm{N}^{\circ} 23214,1994\right) \text {. } \\
\end{array}$ & $\begin{array}{l}\text { Enterolobium cyclocarpum, } \\
\text { Cordia alliodora, Guazuma } \\
\text { ulmifolia, Ceiba pentandra, } \\
\text { Magnolia poasana, Genipa } \\
\text { americana, Carapa sp, Myro- } \\
\text { xylon balsamum, Quercus spp. }\end{array}$ \\
\hline
\end{tabular}


Es importante, que para el distanciamiento de siembra se consideren las características de las especies como el crecimiento en altura y el diámetro de la copa, por lo que este puede variar en cada caso.

Por otra parte, es recomendable reforestar todas aquellas zonas que presenten altas pendientes, para esto se deben seleccionar especies de porte pequeño o mediano, ya que un árbol de porte grande constituirá mucho peso para este tipo de terreno, debido a que es altamente vulnerable a la erosión y al deslizamiento (Sánchez et al., 2015, p.45).

Las especies arbóreas recomendadas son Bursera simaruba, Byrsonima crassifolia, Calliandra sp, Croton draco, Diphysa americana, Guazuma ulmifolia, Mauria heterophylla, Muntingia calabura, Psidium guajaba, Senna sp. Para las actividades propuestas se recomienda realizar podas de formación, de mantenimiento y sanitarias durante los primeros 3 años (Sánchez et al., 2015, p. 55).

Por otra parte, en lo que respecta al uso de zonas urbanizadas, se recomiendan de igual forma buenas prácticas de uso del suelo como se muestra en el cuadro 10, con el objetivo de mejorar y restablecer la conectividad de las áreas verdes a lo largo del corredor. 
Cuadro 10. Buenas prácticas recomendadas para el uso en zonas urbanizadas.

\begin{tabular}{|c|c|c|c|}
\hline $\begin{array}{l}\text { Tipo de } \\
\text { uso }\end{array}$ & $\begin{array}{l}\text { Actividad } \\
\text { propuesta }\end{array}$ & Procedimiento y recomendaciones & Especies recomendadas \\
\hline \multirow{3}{*}{ 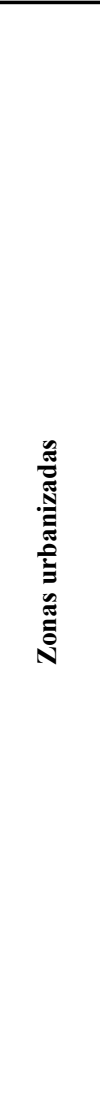 } & $\begin{array}{l}\text { Plantar árboles en } \\
\text { aceras y avenidas. }\end{array}$ & $\begin{array}{l}\text { Se recomienda que los árboles al mo- } \\
\text { mento de plantarlos tengan una altura } \\
\text { mínima de } 1,5 \mathrm{~m} \text { y } 5 \mathrm{~cm} \text { de diámetro en } \\
\text { la base. El fuste debe verse recto y sin } \\
\text { ramas, además, este debe estar libre de } \\
\text { cualquier daño fitosanitario. Las especies } \\
\text { seleccionadas no deben de crecer más de } \\
10 \mathrm{~m} \text { (Sánchez et al, 2015, p. 46). }\end{array}$ & $\begin{array}{l}\text { Malpighia glabra, Ardisia } \mathrm{sp}, \\
\text { Posoqueria latifolia, Sanchezia } \\
\text { parvibracteata, Eugenia uniflo- } \\
\text { ra, Roupala montana, Croton } \\
\text { niveu, Plumeria rubra, Ligus- } \\
\text { trum lucidum, Callistemon } \mathrm{sp}, \\
\text { Bauhinia purpurea. }\end{array}$ \\
\hline & $\begin{array}{l}\text { Reforestar parques } \\
\text { urbanos y/o susti- } \\
\text { tución por especies } \\
\text { idóneas. }\end{array}$ & $\begin{array}{l}\text { Se recomienda que los árboles al mo- } \\
\text { mento de plantarlos tengan una altura } \\
\text { mínima de } 1,5 \mathrm{~m} \text { y } 5 \mathrm{~cm} \text { de diámetro en } \\
\text { la base. En estos casos se podrán selec- } \\
\text { cionar árboles de porte grande, con fustes } \\
\text { gruesos y copas amplias o compactas, } \\
\text { siempre que los individuos tengan su- } \\
\text { ficiente espacio para desarrollarse sin } \\
\text { peligro de afectar infraestructura. Será } \\
\text { necesario escoger individuos de impor- } \\
\text { tancia ecológica con frutos y flores como } \\
\text { atractivo para fauna silvestre (Sánchez et } \\
\text { al, 2015, p. 46). }\end{array}$ & $\begin{array}{c}\text { Malpighia glabra, Trichilia } \\
\text { havanensis, Ardisia sp, Tabe- } \\
\text { buia ochracea, Tabebuia rosea, } \\
\text { Posoqueria latifolia, Roupala } \\
\text { montana, Diphysa americana, } \\
\text { Genipa americana, Plumeria } \\
\text { rubra, Psidium guajava, Ficus } \\
\text { sp, Eriobotrya Japonica, Cojo- } \\
\text { ba arbore, Mauria heterophyll, } \\
\text { Jacaranda caucan, Diloden- } \\
\text { dron sp. }\end{array}$ \\
\hline & $\begin{array}{c}\text { Restaurar las áreas } \\
\text { degradadas de las } \\
\text { riberas de ríos. }\end{array}$ & $\begin{array}{l}\text { Seleccionar especies que se adecuen me- } \\
\text { jor al bosque ripario o de galería, además } \\
\text { se debe considerar si los árboles estarán } \\
\text { expuestos al tránsito de personas por lo } \\
\text { que pueden sufrir daños, en estos casos } \\
\text { es recomendable plantar árboles con una } \\
\text { altura mínima de } 1,5 \mathrm{~m} \text {. El porte máximo } \\
\text { sugerido para los individuos debe ser me- } \\
\text { nor a } 15 \mathrm{~m} \text { y que sean árboles con buen } \\
\text { atractivo (flores o frutos) (Subcomisión } \\
\text { Heredia - CGICRG Tárcoles, sf). }\end{array}$ & $\begin{array}{c}\text { Luehea speciosa, Ochroma } \\
\text { pyramidale, Stemmadenia } \\
\text { donnell-smithii, Urera } \mathrm{sp}, \\
\text { Bursera simaruba, Gliricidia } \\
\text { sepium, Cecropia } \mathrm{sp}, \text { Inga } \mathrm{sp}, \\
\text { Zygia longifolia, Clusia rosea, } \\
\text { Ficus sp, Guazuma ulmifoli, } \\
\text { Cassia grandis, Acnistus } \\
\text { arborescens. }\end{array}$ \\
\hline
\end{tabular}

\section{Fragmentación de la cobertura forestal}

La figura 7 muestra el grado de fragmentación de la cobertura forestal presente en el CBIRT, donde se obtuvieron cuatro niveles de segmentación, los mayores porcentajes corresponden a altamente fragmentado (46,61\%) y fragmentación moderada $(45,8 \%)$, por otra parte, en menor proporción se encuentran insularizado $(4,17 \%)$ y sin fragmentación $(3,42 \%)$. 
Figura 7. Grado de fragmentación de la cobertura forestal en el Corredor Biológico Interurbano Río Torres, San José, Costa Rica.

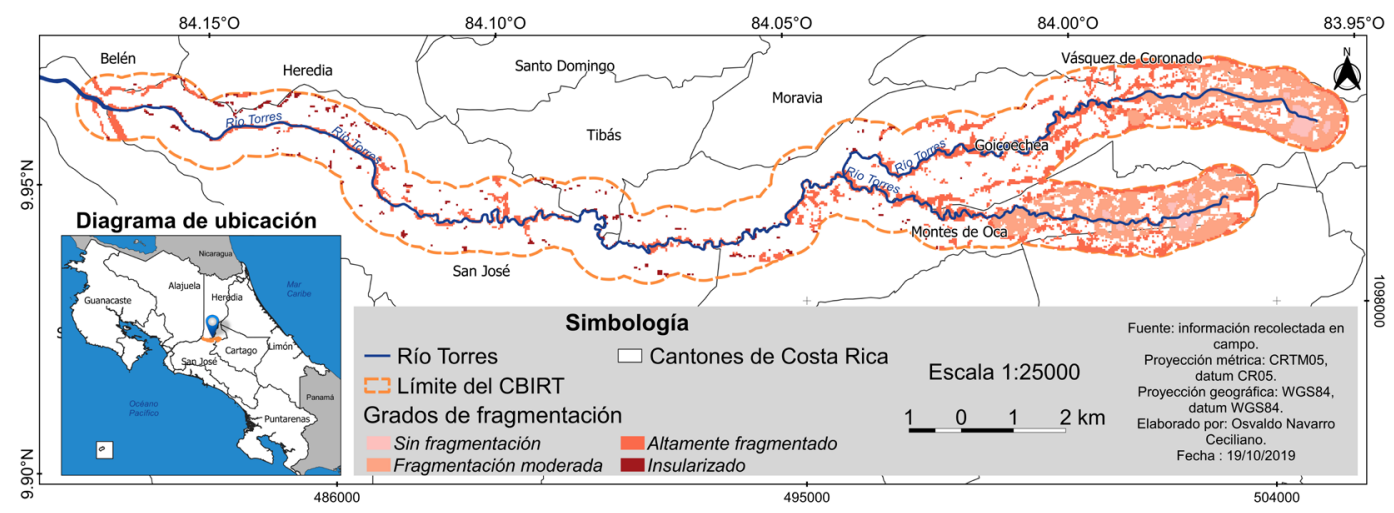

El CBIRT ha sufrido una seria segmentación de la cobertura forestal, esto se observa en las zonas media y baja del corredor, las cuales poseen un alto grado de fragmentación causado principalmente por la necesidad de establecer nuevos usos del suelo, lo que conlleva a cambios en la composición y estructura del bosque provocando afectación en la biodiversidad existente (Trapp, Day, Flaherty, Zollner, \& Smith, 2019, p. 44), sin embargo, es importante destacar que la zona alta posee menor grado de fragmentación.

Para restablecer la conectividad de la cobertura forestal a lo largo del corredor se recomienda intervenir con mayor prioridad las áreas a orillas del río que presenten un grado de fragmentación insularizado, normalmente se consideraría que este grado no es apto para restablecer conexión debido al nivel de aislamiento que poseen (Gastezzi-Arias, Alvarado-García, y Pérez-Gómez, 2016, p. 40) (Rico, 2017, p. 29).

Sin embargo, el objetivo principal es permitir el paso e interacción de las diferentes especies entre la parte alta, media y baja del corredor. Además, es fundamental conectar las áreas que poseen el grado altamente fragmentado, considerando las rutas más factibles que mejoren la integridad ecológica y la conservación de los recursos naturales (Reza, Abdullah, Nor \& Ismail, 2018, p. 361) esto debido a que gran proporción de la cobertura forestal del corredor posee esta condición. 
En el caso de los sitios que presentan una fragmentación moderada, al tener un menor grado de aislamiento, no requieren la implementación inmediata de las actividades recomendadas en este estudio sin embargo, en la medida de lo posible debe procurarse mejorar la conectividad para no alcanzar mayores niveles de fragmentación y asesorar a los propietarios y encargados de estas áreas con el fin de concientizar sobre su importancia para la conservación de la biodiversidad y los servicios ecosistémicos que ofrecen (Naime, Mora, Sánchez-Martínez, Arreola y Balvanera, 2020, p. 2).

Con respecto a los parches de bosque que se encuentran sumamente aislados y que no forman parte de la trama principal de conexión del corredor, se recomienda implementar las estrategias de conectividad a largo plazo.

\section{Conclusiones}

La categoría de capacidad de acogida más representativa es agricultura sin limitaciones, con 79,75\%, mientras que la menos representativa es agroforestería con cultivos anuales, con $0,02 \%$, lo que quiere decir que el CBIRT se caracteriza por pertenecer a categorías de capacidad de acogida poco limitantes de uso del suelo.

Al considerar las zonas de protección para determinar la capacidad de acogida del suelo, la categoría más representativa cambia a tierras forestales de protección con 2338,13 hectáreas $(54,2 \% \%$ ) y la segunda más representativa es agricultura sin limitaciones con 1787,77 hectáreas $(41,44 \%)$, por lo tanto, el CBIRT se categoriza en zonas con altas y mínimas limitaciones.

En lo que respecta al uso del suelo se obtuvieron cinco clases, el mayor porcentaje de área corresponde a la clase zonas urbanizadas con un $53,62 \%$, seguido de la cobertura forestal con $25,7 \%$, ganadería y pastos con $19,91 \%$, agricultura $0,75 \%$ y en menor proporción zonas húmedas con $0,02 \%$. Aunado a esto para la clasificación se obtuvo una exactitud global de $93,75 \%$. En cuanto al sobreuso de suelo en el CBIRT, se determinó que 640,27 hectáreas se encuentran en sobreuso, de este valor el 95,17\% corresponde a ganadería y pastos y $4,82 \%$ a agricultura.

Las prácticas definidas en este estudio para mejorar las condiciones de uso del suelo en el CBIRT abarcan actividades de reforestación y/o restauración, procedimientos y recomendaciones y posibles especies a utilizar. Con el estudio se evidencia que un $96,58 \%$ de la cobertura forestal 
posee algún grado de fragmentación, sin embargo, solo un 4,17\% del total está representado por el nivel más crítico de aislamiento (insularizado).

\section{Recomendaciones}

Se debe realizar un monitoreo continuo para detectar posibles cambios en el uso del suelo, con el objetivo de mantener un mayor control del uso óptimo del mismo y prevenir la pérdida de cobertura forestal.

Se recomienda que las actividades propuestas sean implementadas a corto plazo en las zonas que presentan un grado de fragmentación insularizado, dando prioridad a los sitios que se ubican a lo largo del río Torres, a fin de garantizar la conectividad de la cobertura forestal que se encuentra aislada.

Es importante aumentar la cobertura forestal del sitio por lo cual se recomienda implementar las prácticas propuestas en este estudio, a fin de mejorar la calidad de vida de los habitantes del corredor y procurar la protección y conservación de la biodiversidad presente.

\section{Referencias}

Acosta, J., Suango, V., Proaño, J. y Zambrado, J. (septiembre, 2016). Determinación de la capacidad de acogida por medio de la zonificación paisajística para el Ecuador continental. ResearchGate, 4(1), 34-44. https://doi.org/10.36331/revista.v4i1.24

Adams, VM., Pressey, R. L. \& Álvarez-Romero, JG. (junio, 2016). Using Optimal Land-Use Scenarios to Assess Trade-Offs between Conservation, Development, and Social Values. PLOS ONE, 11(6), 1-20. https://doi.org/10.1371/journal.pone.0158350

Arjomandi, A., Mortazavi, S., Khalilian, S. \& Garizi, A. (enero, 2021). Optimal land-use allocation using MCDM and SWAT for the Hablehroud Watershed, Iran. ScienceDirect, 100(104930), 1-11. Recuperado de https://doi.org/10.1016/j.landusepol.2020.104930

Astola, H., Häme, T., Sirro, L., Molinier, M. \& Kilpi, J. (marzo, 2019). Comparison of Sentinel-2 and Landsat 8 imagery for forest variable prediction in boreal region. ScienceDirect, 223, 257-273. Recuperado de: https://doi.org/10.1016/j.rse.2019.01.019

Boca, T. y Rodríguez, G. (2012). Métodos estadísticos de la evaluación de la exactitud de productos derivados de sensores remotos. Recuperado 
Osvaldo Navarro-Ceciliano

Estudio de capacidad de acogida del suelo en el Corredor Biológico Interurbano Río Torres, Costa Rica

de https://inta.gob.ar/documentos/metodos-estadisticos-de-la-evaluacion-de-la-exactitud-de-productos-derivados-de-sensores-remotos

Casamor, J. y Calafat, A. (octubre, 2018). El problema del aterramiento de embalses y el cálculo de las reservas hidráulicas. Tierra y Tecnología, 52. Recuperado de http://dx.doi.org/10.21028/jlc.2018.10.01

Casco, G., Arias, L., Mareco, H., Kindgard., A. (2016). Informe final de evaluación de exactitud temática del mapa de cobertura forestal y cambio de uso de la tierra para los años 2000, 2005 y 2011. Recuperado de http://www.infona.gov.py/application/files/6514/7405/3096/ Evaluacion_exactitud_Mapas_000511_160516.pdf

Feoli-Boraschi, S. (septiembre, 2012). Corredores biológicos: una estrategia de conservación en el manejo de cuencas hidrográficas. Revista Forestal Mesoamericana kurú, 6(17), 1-5. Recuperado de https://revistas.tec.ac.cr/index.php/kuru/article/view/385

Galacho, F. y Arrebola, J. (julio-diciembre, 2013). Modelo de evaluación de la capacidad de acogida del territorio con SIG y técnicas de decisión multicriterio respecto a la implantación de edificaciones en espacios rurales. Investigaciones Geográficas, 60, 69-85. https://doi. org/10.14198/INGEO2013.60.04

Gastezzi-Arias, P., Alvarado-García, V. y Pérez-Gómez, G. (2016). La importancia de los ríos como corredores interurbanos. Revista Biocenosis, 31(1-2), 39-45. Recuperado de https://investiga.uned.ac.cr/ revistas/index.php/biocenosis/article/view/1725/1952

Gómez, S. C. (2014). Metodología para el estudio de la capacidad de acogida del suelo en cuencas hidrográficas (Tesis de Maestría, Universidad de Cuenca). Recuperado de http://dspace.ucuenca.edu.ec/ handle/123456789/5600

GRUPO G6. (2018). Sistema de Monitoreo de la Cobertura y Uso de la Tierra y Ecosistemas (SIMOCUTE). Sistema de clasificación del uso y la cobertura de la tierra para Costa Rica. Versión 1.1. San José, Costa Rica.

ICE. (2019). Página principal, Quiénes somos, Historia. Recuperado de https://www.grupoice.com/wps/portal/ICE/quienessomos/ quienes-somos/historia

Ley Forestal $N^{\circ} 7575$. (1996). Recuperado de http:/www.pgrweb.go.cr/scij/ Busqueda/Normativa/Normas/nrm_texto_completo.aspx?param1=NRT C\&nValor1=1\&nValor2=41661\&nValor3=94526\&strTipM=TC (1996). 
Luther, D.A., Cooper, W.J., Wolfe, J.D., Bierregaard, R.O., Gonzalez, A. \& Lovejoy, T.E. (septiembre, 2020). Tropical forest fragmentation and isolation: Is community decay a random process? ScienceDirect, 23(e01168), 1-9. Recuperado de https://doi.org/10.1016/j.gecco.2020.e01168

McDowell, RW., Snelder, T., Harris, S., Lilburne, L., Larned, S T., Scarsbrook, M., Curtis, A., Holgate, B., Phillips, J. \& Taylor, K. (Agosto, 2018). The land use suitability concept: Introduction and an application of the concept to inform sustainable productivity within environmental constraints. ScienceDirect, 91, 212-219. Recuperado de https://doi.org/10.1016/j.ecolind.2018.03.067

Metodología Determinación Capacidad Uso Tierras Costa Rica, Decreto $N^{\circ}$ 41960-MAG-MINAE. (2019). Consultado el 17 de agosto del 2019. Recuperado de http://www.pgrweb.go.cr/scij/Busqueda/Normativa/Normas/nrm_texto_completo.aspx?param $1=$ NRTC\&nValor $1=1 \&$ nValor $2=90001 \&$ nValor $3=118346 \&$ param $2=1 \&$ strTipM $=$ TC $\& 1$ Resultado $=1 \& \operatorname{strSim}=\operatorname{sim} p$

Naime, J., Mora, F., Sánchez-Martínez, M., Arreola, F. y Balvanera, P. (octubre, 2020). Economic valuation of ecosystem services from secondary tropical forests: trade-offs and implications for policy making. ScienceDirect, 473(118294), 1-10. Recuperado de https://doi. org/10.1016/j.foreco.2020.118294

Organización Internacional del café. (2019). Informe del mercado del café. Recuperado de http://www.ico.org/documents/cy2018-19/cmr0719-c.pdf

FAO (Organización de las Naciones Unidas para la Alimentación y la Agricultura). (2018). Guía de buenas prácticas para la gestión y uso sostenible de los suelos en áreas rurales. Recuperado de: https://www.minambiente.gov.co/images/ AsuntosambientalesySectorialyUrbana/pdf/suelo/Guia_de_buenas_practicas_para_la_gestion_y_uso_sostenible_de_los_suelos_en_areas_rurales.pdf?fbclid=IwAR1KCrORwDHbSpn3BOjjyEDAnGZxu3_17-MXyDhEWZs5hHa8N3pDFgyNGg

Pérez-Gómez, G., Gastezzi-Arias, P. y Vega-Quesada, A. (noviembre, 2016). Avifauna poco frecuente en la microcuenca del río Torres, San José, Costa Rica. Zeledonia, 20(2), 20-27. Recuperado de https://www.zeledonia.com/uploads/7/0/1/0/70104897/zelnov2016-20-27.pdf 
Osvaldo Navarro-Ceciliano

Estudio de capacidad de acogida del suelo en el Corredor Biológico Interurbano Río Torres, Costa Rica

Proyecto Biodiver_City San José. (2020). Página principal, Corredores Biológicos Interurbanos, Corredor Biológico Interurbano Río Torres. Recuperado de http://biocorredores.org/biodiver-city-sanjose/ corredores-biologicos/corredor-biologico-interurbano-rio-torres-0

Reza, M. I., Abdullah, S. A., Nor, S. B., \& Ismail, M. H. (abril, 2018). Landscape Pattern and Connectivity Importance of Protected Areas in Kuala Lumpur Conurbation for Sustainable Urban Planning. International Journal of Conservation Science, 9(2). 361-372. Recuperado de http://web.a.ebscohost.com.ezproxy.itcr.ac.cr/ehost/ pdfviewer/pdfviewer?vid=3\&sid=e0bcf904-db84-47ab-825e0c7655ac7fca\%40sessionmgr4007

Reglamento a la Ley de Biodiversidad $N^{\circ}$ 34433. (2008). Artículo 3. Consultado el 20 de marzo de 2019. Recuperado de http://www.pgrweb. go.cr/scij/Busqueda/Normativa/Normas/nrm_texto_completo.aspx? param $1=$ NRTC $\&$ nValor $1=1 \&$ nValor $2=62838 \&$ nValor $3=110325 \&$ st rTipM=TC

Rico, Y. (agosto, 2017). La conectividad del paisaje y su importancia para la biodiversidad. ResearchGate, 34, 28-30. Recuperado de https:// www.researchgate.net/publication/321197535_La_conectividad_ del_paisaje_y_su_importancia_para_la_biodiversidad

Sánchez, G., Valle D., Scorza, F., Feoli, S. y Ärtavia, R. (2015). Protocolo de Reforestación para la Rehabilitación y Mantenimiento en Áreas de Protección de la GAM. Recuperado de http://www.edumovil. com/conectividad_ecosistemas/wp-content/uploads/2017/11/Protocolo-Reforestacion-Rehab-Mant-de-AP-en-la-GAM.pdf

Sánchez, G., Valle-Hidalgo, D., Feoli, S. y Murillo, J. (2016). Perfil del Corredor Biológico Interurbano Río Torres Reserva de la Biosfera. Recuperado de http://www.edumovil.com/conectividad_ecosistemas/wp-content/uploads/2017/11/Perfil_CBI-RT-RB_02Mar-2017.pdf

Sistema Nacional de Áreas de Conservación [SINAC]. (2018). Plan Estratégico 2018-2025 del Programa Nacional de Corredores Biológicos de Costa Rica (Informe Final). Recuperado de https://enbcr.go.cr/ sites/default/files/sinac_2018_planestrategico_programa_nacional_ de_corredores_biologicos_costa_rica.pdf 
Subcomisión Heredia-CGICRG Tárcoles. (s.f). Protocolo de recuperación de áreas de protección y arborización para la provincia de Heredia. Recuperado de https://www.belen.go.cr/documents/20181/76421/Protocolo+Reforestaci\%C3\%B3n+Heredia/ c6e7d54d-f5ac-4bcb-bde5-676eb3051bf6

Suryatmojo, H. (2014). Recovery of Forest Soil Disturbance in the Intensive Forest Management System. ScienceDirect, 20, 832-840. doi: https://doi.org/10.1016/j.proenv.2014.03.101

Thakkara, A.K., Desai, V.R., Patel, A. \& Potdar, M.B. (junio, 2017). Postclassification corrections in improving the classification of Land Use/Land Cover of arid region using RS and GIS: The case of Arjuni watershed, Gujarat, India. ScienceDirect, 20(1), 79-89. Recuperado de https://doi.org/10.1016/j.ejrs.2016.11.006

Trapp, S., Day, C., Flaherty, E., Zollner, P., \& Smith, W. (febrero, 2019). Modeling impacts of landscape connectivity on dispersal movements of northern flying squirrels (Glaucomys sabrinus griseifrons). ScienceDirect, 394, 44-52. Recuperado de https://doi.org/10.1016/j. ecolmodel.2018.12.025

Trujillo-Acosta, A., Peraza-Estrella, M., Marina-Hipólito, J. y Feoli, S. (diciembre, 2016). Evaluación del Corredor Interurbano Río Torres, Costa Rica. Revista Forestal Mesoamérica Kurú, 14(34), 53-62. https://doi.org/10.18845/rfmk.v14i34.3001

Viscarra, R.A., Behrens, T., Ben-Dor, E., Brown, D.J., Demattêe, J.A., Shepherd, K.D. Shig, Z. Stenberg, B., Stevens, A., Adamchuk, V., Aïchi, H., Barthès, B.G., Bartholomeus, H.M. Bayer, A.D., Bernou, M., Böttcher, K., Brodský, L., Du, C.W., \& Ji, W. (abril, 2016). A global spectral library to characterize the world's soil. ScienceDirect, 155, 198-230. Recuperado de https://doi.org/10.1016/j. earscirev.2016.01.012

Zweifel, L., Meusburger, K. \& Alewell, C. (diciembre, 2019). Spatiotemporal pattern of soil degradation in a Swiss Alpine grassland catchment. ScienceDirect, 235, 1-11. Recuperado de https://doi. org/10.1016/j.rse.2019.111441 